\title{
Does understanding metrological traceability in analytical measurement require going "back to basics"?
}

\author{
Paul De Bièvre
}

Published online: 26 February 2014

(C) Springer-Verlag Berlin Heidelberg 2014

Traceability of a measurement result to a common reference is termed 'metrological traceability' in the International vocabulary of metrology, VIM [1], entry 2.41. It is a concept of basic importance because metrological traceability is a precondition to 'metrological comparability of measurement results' (entry 2.46 in [1]), and comparing measurement results is a major goal of measurement. Comparing measurement results, which are not traceable to a common reference, is a meaningless operation. Sadly enough, the term traceability was not really well understood in the analytical measurement community until about 20 years ago (the first Workshop on "comparability" and "traceability" was held at IRMM in Geel, Belgium in November 1992). Pictures of 'metrological traceability' of analytical measurement results have been shown in the form of a 'metrological traceability chain' (entry 2.42 in [1]), for instance in an IUPAC study released in 2011 [2].

Yet, the concept and associated term metrological traceability are still giving rise to complicated reasonings, confusing descriptions, and erroneous conclusions. Is it indeed so difficult? Let us try to improve understanding of "metrological traceability."

Usually, a measurement involves the measurement of a 'certified reference material, CRM' (entry 5.14 in [1]), i.e., a 'reference material, RM' (entry 5.13 in [1]) in which a known quantity or property value with associated uncertainty is embodied. To calibrate the measurement, we like

Disclaimer The author is a member of the Joint Committee on Guides for Metrology (JCGM), Working Group 2 (VIM). The opinions expressed in this Column do not necessarily represent the view of the Working Group or of ACQUAL.

P. De Bièvre $(\bowtie)$

Kasterlee, Belgium

e-mail: paul.de.bievre@skynet.be to choose a CRM whose certified value is as close as possible to the expected measured value (entry 2.10 in [1]) of the test sample. We even like the CRM to have a chemical structure ("matrix") as similar as possible to the test material structure in which the analyte is embodied.

Note: an RM, hence also a CRM (a special case of an RM) can only serve one purpose in one and the same measurement: either being used as a calibrator, or as a trueness control material (entry 5.13 Note 2 in [1]). In our discussion here, we only consider the first of these two functions: using it as a calibrator.

What then is a "reference" needed for the definition of metrological traceability? The answer is given in Note 1 of entry 2.41 in VIM [1]:

1. A measurement unit through its practical realization ("measurement unit" here means "definition of the unit")

Note a: The definition of a measurement unit is not measured thus having measurement uncertainty zero; however, its practical realization has a measurement uncertainty because it results from work in the laboratory.

Note b: Metrological traceability is possible to other references than (realizations of) SI units.

2. A measurement standard

Let us look at once to two examples of measurement standards from this entry, Examples 5 and 6 quoted hereafter:

- Set of reference solutions of cortisol having a certified quantity value with measurement uncertainty for each solution;

\footnotetext{
${ }^{1}$ Single quotes are used to designate certain VIM concepts [1] at their first usage in this Column.
} 
- Reference material providing quantity values with measurement uncertainties for the mass concentrations of ten different proteins.

The entry provides several other examples in physics; all the examples constitute the "family" of measurement standards. When a certificate issued by an authoritative body, accompanies a measurement standard, stating the embodied one or more quantity values with associated uncertainty, it is known as "CRM." It can serve the purpose of calibrating in which case it is a link of any metrological traceability chain. Several examples of such chain are, e.g., demonstrated in [2].

\section{A measurement procedure}

If a sufficiently comprehensive description of the measurement is contained in a 'measurement procedure' (entry 2.6 in [1]) -which is a document containing a detailed description- it can serve the purpose of being a "reference" for realizing a quantity.

What do these "references" offer us?

1. We will not treat this case here, but possibly in a future Column if found useful.

2. The use of the measurement standard called "CRM" to establish metrological traceability to, is a well-known practice among analysts, but it is true that not all of the CRM providers indicate further details of the metrological traceability to a "higher" reference, of the certified value with a stated uncertainty in their CRM Certificates or accompanying documents (so far). But, the quantity value embodied in a CRM can serve as a common reference for measurements of the same measurand performed by different analysts at different times, different locations, and under different circumstances (the CRM quantity value can be accepted as "reference" by the parties involved, e.g., between buyer and seller). The responsibility of the analyst for the metrological traceability of the measured value of his/her measurand ends in the CRM quantity value. And, as indicated above, if different metrological traceability chains of different measurement results demonstrate traceability to that reference for each of the results, then and only then, these results become comparable.

Note: CRM users buying CRMs are therefore encouraged to buy (and pay for) values with indicated metrological traceability and associated measurement uncertainty, rather than just buy (and pay for) a "material" [3].

When purchased, CRMs must be accompanied by a certificate from an authoritative body and are the "realization of the definition of a given quantity, with stated quantity value and associated measurement uncertainty, ..." (entry 5.1 in [1]). For example, a mass fraction of substance $\mathrm{X}$ in matrix $\mathrm{Y}$, expressed as mass per mass, with stated quantity value (the value of the specified mass fraction) and associated measurement uncertainty (the uncertainty of the certified mass fraction).

The CRM can also be substituted in a number of cases by "synthesizing" a value. An example of that is the preparation of a synthetic solution of, say, a known mass (obtained by weighing) of glucose of known purity in a known mass (idem) of pure water (aimed at preparing a solution to calibrate a "glucose meter"). This makes this solution into a "primary measurement standard' (entry 5.4 in [1]) because it has been prepared by using a 'primary reference measurement procedure' (entry 2.8 in [1]). Such a procedure is a "measurement procedure used to obtain a measurement result without relation to a measurement standard for a quantity of the same kind." In this example that means: without directly measuring the glucose mass fraction in the solution, but "synthesizing" the certified value. The quantity value embodied in this measurement standard can now be expressed as a dimensionless ratio: the ratio of two mass values. By virtue of meeting the requirements in the definition of primary measurement standard, it can serve as a "reference" for metrological traceability.

3. The measurement procedure, i.e., a detailed description of a measurement, enables to define an "operationally defined measurand." It is a useful possibility when the measurand cannot yet be defined independently from the procedure. That is still frequently the case and will stay as such for measuring functional properties (in material science, physics, chemistry, biology, ...) such as measuring, e.g., the activity of an enzyme which is an indicator of upcoming cardiovascular troubles. In matters of "uncertainty," any measured value obtained by such a procedure, can only be equipped by a statement of reproducibility (or repeatability) and robustness of its results, possibly increased by an evaluation of the limited amount of information about the measurand.

A rather simple example of a measurement procedure, leading to an operationally defined measurand, is the measurement of "leachable Cd mass fraction" of a ceramic plate, i.e., the $\mathrm{Cd}$ content expressed in $\mathrm{mmol} / \mathrm{kg}$, of a specified acid solution having been in contact with a specified plate for a given time and a given temperature. 
We conclude the following:

There are three references which go with the definition of metrological traceability. That can be the definition of a measurement unit, i.e. a value without a measurement uncertainty, a CRM embodying a value with an associated uncertainty, and a measurement procedure, with an approximate (and incomplete) evaluation of uncertainty.

Is it helpful for the understanding of metrological traceability in analytical measurement to go "back to basics"? Hopefully.

As usual, any comment, question, or amendment is welcome, preferably as a contribution to the Discussion Forum of this Journal.

\section{References}

1. BIPM, IEC, IFCC, ILAC, IUPAC, IUPAP, ISO, OIML. The international vocabulary of metrology - basic and general concepts and associated terms (VIM), 3rd edn. JCGM 200:2012 at http:// www.bipm.org/vim

2. De Bièvre P, Fajgelj A, Dybkaer R, Hibbert B (2011) Metrological traceability of measurement results in chemistry: concepts and implementation IUPAC Recommendations. Pure Appl Chem 83:1873-1935, Section 9 at http://iupac.org/publications/pac/83/ $10 / 1873$

3. De Bièvre P (2000) When we purchase a "reference materiel", what do we pay for? Accred Qual Assur 5:307 